\title{
Evaluating Bone Loss with Bone Turnover Markers Following Acute Spinal Cord Injury
}

\author{
Prince Thakkar ${ }^{1}$, Naveen B. Prakash ${ }^{1}$, George Tharion ${ }^{1}$, Sahana Shetty ${ }^{2}$, \\ Thomas V. Paul ${ }^{2}$, Joseph Bondu ${ }^{3}$, Bijesh Yadav ${ }^{4}$ \\ ${ }^{1}$ Department of Physical Medicine and Rehabilitation, Christian Medical College, Vellore, India \\ ${ }^{2}$ Department of Endocrinology, Christian Medical College, Vellore, India \\ ${ }^{3}$ Department of Clinical Biochemistry, Christian Medical College, Vellore, India \\ ${ }^{4}$ Department of Biostatistics, Christian Medical College, Vellore, India
}

Study Design: Prospective observational study.

Purpose: To evaluate bone turnover markers (BTMs) in individuals with acute spinal cord injury (SCI) and to compare the results with those of healthy controls and postmenopausal females.

Overview of Literature: SCI significantly impacts bone health. Change in bone mineral density appears 6 months after SCl and rapid bone loss during the acute phase is often underestimated, resulting in osteoporosis and a high risk of sublesional fractures. However, few studies have evaluated BTMs in the Indian SCI population. Despite a high risk of fracture, there are no guidelines for the diagnosis, monitoring, and management of SCl-induced osteoporosis.

Methods: Twenty patients within 1 month of traumatic SCI who had been admitted to a tertiary care rehabilitation center were included in this study. Serum BTMs, C telopeptide (CTX) as a bone resorption marker, and osteocalcin as a bone formation marker, were serially measured at baseline, and 3 and 6 months after SCI. BTMs of SCI patients were compared with those of a control group of age-matched healthy males, premenopausal females, and a vulnerable group of postmenopausal females.

Results: BTMs were significantly elevated in patients with SCl, with maximum levels observed at the 3rd month of injury. At baseline, the bone resorption marker CTX was approximately 3 times higher in SCl patients than in the control male population and premenopausal females, and about double that of postmenopausal females. The rise in the bone formation marker was marginal in comparison to that of the bone resorption marker. BTMs were persistently elevated and did not reach the normative range until the 6th month of SCl.

Conclusions: Raised bone resorption markers in comparison to bone formation markers indicate hyper-resorption-related bone loss following acute SCl. Markedly elevated bone resorption markers in the SCI population, compared with those in control and vulnerable groups, emphasize the need for early bone health monitoring and management.

Keywords: Spinal cord injuries; Bone remodeling; Bone turnover markers; C telopeptide; Osteocalcin; Osteoporosis

Received Jan 6, 2019; Revised Apr 23, 2019; Accepted May 20, 2019

Corresponding author: Prince Thakkar

Department of Physical Medicine and Rehabilitation, Christian Medical College, Vellore, Pin-632004 Tamil Nadu, India

Tel: +91-04162282158, Fax: +91-04162282158, E-mail: princethakkar123@gmail.com 


\section{Introduction}

Spinal cord injury (SCI) significantly impacts bone health, eventually leading to complications such as osteoporosis and pathological fractures $[1,2]$. The rapid and extensive decline in bone density following SCI is greater than that seen upon immobilization alone [3]. Skeletal tissue below the neurological level of injury undergoes rapid remodeling following SCI, although a precise understanding of the pathophysiology of osteoporosis has yet to be obtained [4]. Factors responsible for bone loss and osteoporosis in SCI patients include mechanical unloading, neural denervation, disuse osteoporosis, changes in the osteoprotegerinRANKL system, and altered Wnt signaling pathways $[5,6]$. Changes in bone metabolism appear within a few days of injury and peak at about 6 months, followed by a continuous decrease in bone mass of about $1 \%-5 \%$ per year extending up to 6-8 years [7]. Cancellous and cortical bone has been observed to undergo about 5\%-50\% regional bone loss in the sublesional distal extremities, primarily the distal femur and proximal tibia [8,9]. Bone loss and osteoporosis appear to be inevitable sequelae in all complete SCI patients, which predisposes them to lowenergy fractures. The diagnosis of osteoporosis is also often delayed in SCI patients until pathological fractures are encountered [10]. Hence, early assessments of bone loss and fracture-related risk factors are essential for the prevention of osteoporosis and fragility fractures in the SCI population. Bone mineral density (BMD) assessment using dual-energy X-ray absorptiometry (DEXA) scanning is the current gold standard test for the diagnosis of osteoporosis. However, conventional DEXA scanning, measuring $\mathrm{BMD}$ at the lumbar spine and proximal femur, may not be an ideal tool in the SCI population as BMD loss differs at distal and proximal epiphysis of trabecular bones in patients with SCI [8]. DEXA scanning has its own limitations, being an expensive investigation with limited availability in many parts of India [11]. An alternative is peripheral quantitative computed tomography scanning, which determines both regional BMD and cortical thinning of the diaphysis, but it is also costly and not readily available. Hence, there is a need to estimate the fracture risk using SCI-specific risk factors with and without BMD, using tools such as FRAX (a fracture risk assessment tool) [12]. Bone turnover markers (BTMs) can potentially be used for fracture risk assessment as they respond earliest and reflect the underlying bone turnover process following spinal injury. A cost-effective and reliable assay of BTMs is now available with improved specificity and sensitivity. However, studies evaluating BTMs in the Indian SCI population are limited. To our knowledge, this is the first study in the Indian population evaluating BTMs in acute SCI and comparing them with those in healthy controls and a vulnerable population such as postmenopausal females.

\section{Materials and Methods}

\section{Study design and participants}

This was a longitudinal observational study conducted at a tertiary care teaching hospital from 1st April, 2014, to 28th February, 2016. This study was approved by the Institutional Review Board and Ethical Committee of Christian Medical College, Vellore, Tamil Nadu, India. (IRB MIN no., 8374, dated 6. 3. 2014). Informed consent was obtained from all the individual participants included in the study. Thirty acute SCI patients aged $18-45$ years presenting within 1 month of traumatic SCI (with a neurological level between T4 and L1) were included in the study and followed over a period of 6 months. Twenty patients completed the study, which comprised 18 males and two premenopausal females. Patients with non-traumatic SCI, deranged renal functions (contracted kidneys in the ultrasound and serum creatinine $>1.5 \mathrm{mg} / \mathrm{dL}$ ), bone disease, under treatment for osteoporosis, regularly taking drugs with a potential adverse impact on bone health (e.g., steroids), hepatic dysfunction, or hyperthyroidism were excluded from the study. A complete medical history, family history, medications, and bone-specific information regarding previous fractures and falls were collected at the initial screening visit. Level and grade of injury were assessed according to the American Spinal Injury Association Impairment Scale (AIS) classification.

Patients with complete injury (AIS-A) have no motor or sensory function below the neurological level of injury, whereas those with incomplete lesions (AIS-B, -C, -D) have sensory and/or motor function preserved with varying degrees of muscle and sensory function below the neurological level of injury [13]. Laboratory evaluations for hepatic and renal function, 25-hydroxyvitamin D (25[OH]D) levels, and thyroidal status were performed at this time. After screening, participants who qualified for the study underwent assessment of serum BTM levels. 
All of the participants with low serum 25(OH)D levels $(<20 \mathrm{ng} / \mathrm{mL}$ ) were supplemented with oral cholecalciferol, at $60,000 \mathrm{IU}$ once a week for 6 weeks, followed by once a month for 6 months along with calcium phosphate at 500 $\mathrm{mg}$ for 6 months. All study subjects underwent a routine SCI physiotherapy protocol of the rehabilitation institute depending on their neurological status and physical ability. The SCI rehabilitation protocol includes $6-8$ weeks of a wheelchair training program and ambulation training with orthotic devices. Patients with good abdominal strength (neurological level, T10 and below) were trained for ambulation with knee ankle foot orthosis and elbow crutches. The study population comprised 20 patients with acute $(<1$ month) traumatic SCI, age-matched healthy males, and historical controls of premenopausal and postmenopausal females.

\section{Biochemical tests}

Serum calcium, phosphorus, creatinine, albumin, and alkaline phosphatase were assessed after overnight fasting as part of the routine clinical evaluation. Biochemical parameters were measured in a fully automated computerized microanalyzer (Hitachi model 911; Boehringer Mannheim, Mannheim, Germany). Intact parathyroid hormone (PTH) was measured by chemiluminescence immunoassay using an ADVIA Centaur XP (Siemens Diagnostics, Tarrytown, NY, USA). 25(OH)D was measured by electro-chemiluminescence immunoassay using an Elecsys E170 (Roche Diagnostics, Indianapolis, IN, USA).

Blood samples for the measurement of serum collagen type 1 cross-linked C telopeptide (CTX), serum osteocalcin $(\mathrm{OCN})$, and bone resorption and formation markers were collected between $7 \mathrm{AM}$ and $10 \mathrm{AM}$ after an overnight fast at 1, 3, and 6 months after SCI. The blood samples intended for use in the assessment of BTMs were stored at $-70^{\circ} \mathrm{C}$ until the assay was performed. Serum CTX and OCN were measured by electro-chemiluminescence immunoassay using an Elecsys E170 (Roche Diagnostics). The measuring range for CTX as described in the insert kit was 10-6,000 pg/mL, with analytical sensitivity of $10 \mathrm{pg} / \mathrm{mL}$ and intra- and interassay coefficient of variation $(\mathrm{CV})$ of $5.5 \%$ and $7.6 \%$, respectively. The manufacturer's reference range was $299 \pm 137 \mathrm{pg} / \mathrm{mL}$ (mean \pm 2 standard deviation [SD]) for premenopausal women and $556 \pm 226 \mathrm{pg} / \mathrm{mL}$ (mean $\pm 2 \mathrm{SD}$ ) for postmenopausal women. The measuring range for $\mathrm{OCN}$ as described in the insert kit was $0.5-300 \mathrm{ng} / \mathrm{mL}$, with analytical sensitivity of $0.5 \mathrm{ng} / \mathrm{mL}$ and intra- and interassay CV of $3.3 \%$ and $3.8 \%$, respectively. The manufacturer's reference range was $21.5 \pm 6.30 \mathrm{ng} / \mathrm{mL}$ (mean $\pm 2 \mathrm{SD}$ ) for premenopausal women and $28.99 \pm 10.02 \mathrm{ng} / \mathrm{mL}$ (mean $\pm 2 \mathrm{SD}$ ) for postmenopausal women. Data were collected to observe the change in the BTMs among SCI patients over a period of 6 months (1st, $3 \mathrm{rd}$, and 6 th months) and statistically analyzed. In addition, serum BTMs of the SCI patients were compared with that of an age-matched male control population, premenopausal females, and postmenopausal females from the urban Indian community [14].

\section{Statistical analysis}

The statistical analyses were performed using IBM SPSS ver. 25.0 (IBM Corp., Armonk, NY, USA). Data was entered using EPIDATA software and screened for outliers and extreme values using Box-Cox plot (for the shape of the distribution). Summary statistics were provided for demographic and clinical characteristics. The distributions of CTX and OCN were checked by creating histograms and QQ plots. Comparison between groups was performed using the Mann-Whitney $U$-test. A $p<0.05$ was considered to be statistically significant.

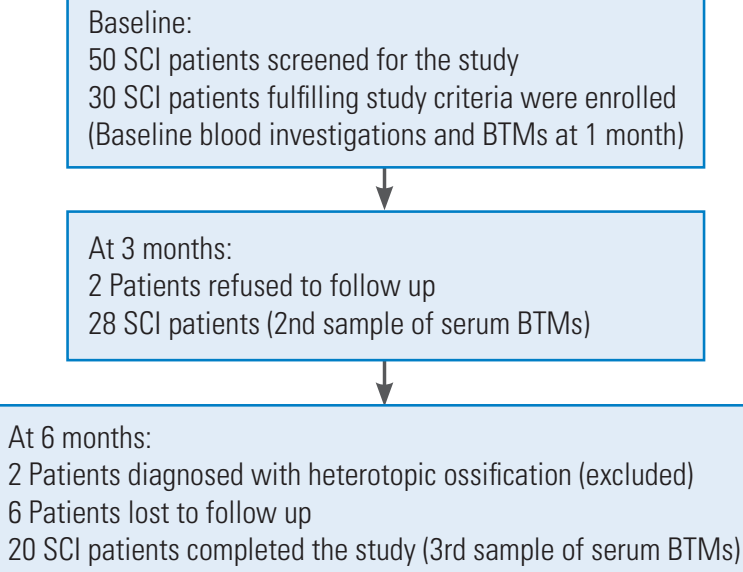

2 Patients diagnosed with heterotopic ossification (excluded)

$20 \mathrm{SCl}$ patients completed the study (3rd sample of serum BTMs)

At 6 months: 6 Patients lost to follow up

Data analysis

$20 \mathrm{SCl}$ patients with baseline, 3rd and 6th month serum BTMs 30 Age-matched healthy males with baseline serum BTMs Historical controls

76 Age-matched premenopausal females with baseline BTMs 76 Postmenopausal females with baseline BTMs

Fig. 1. Study flowchart. SCI, spinal cord injury; BTMs, bone turnover markers. 
Table 1. Mean age, vitamin D, calcium, PTH in acute spinal cord injury and correlation with bone turnover markers

\begin{tabular}{|c|c|c|c|c|c|}
\hline \multirow{2}{*}{ Variable } & \multicolumn{3}{|c|}{$\mathrm{SCl}$} & \multicolumn{2}{|c|}{$p$-value ${ }^{\text {a) }}$} \\
\hline & Male $(n=18)$ & Female $(n=2)$ & Total $(n=20)$ & OCN (ng/mL) & CTX (pg/mL) \\
\hline Age (yr) & $29.11 \pm 6.18$ & $21 \pm 2.82$ & $26.5 \pm 6.39$ & 0.14 & 0.11 \\
\hline Complete injury & 14 & 2 & 16 & 0.42 & 0.06 \\
\hline Incomplete injury & 4 & 0 & 4 & 0.84 & 0.55 \\
\hline PTH (ng/dL) & $16.3 \pm 11.27$ & $3.00 \pm 2.12$ & $15.2 \pm 11.4$ & 0.10 & 0.08 \\
\hline Vitamin D (ng/mL) & $16.5 \pm 10.06$ & $3.09 \pm 0.12$ & $14.99 \pm 10.5$ & 0.95 & 0.11 \\
\hline Calcium (mg/dL) & $8.93 \pm 0.58$ & $9.12 \pm 0.31$ & $8.95 \pm 0.55$ & 0.40 & 0.47 \\
\hline OCN (ng/mL) & $29.57 \pm 11.18$ & $34.23 \pm 5.59$ & $30.04 \pm 10.02$ & - & - \\
\hline CTX (pg/mL) & $1,517.94 \pm 643.04$ & $1,348.333 \pm 349.36$ & $1,500.02 \pm 620.02$ & - & - \\
\hline
\end{tabular}

Values are presented as mean \pm standard deviation or number.

PTH, parathyroid hormone; SCI, spinal cord injury; OCN, osteocalcin; CTX, C telopeptide.

${ }^{a}$ Calculated considering the total SCI population ( $\left.\mathrm{n}=20\right)$; Gender specific $p$-value was not done, as study population had predominant male subjects.

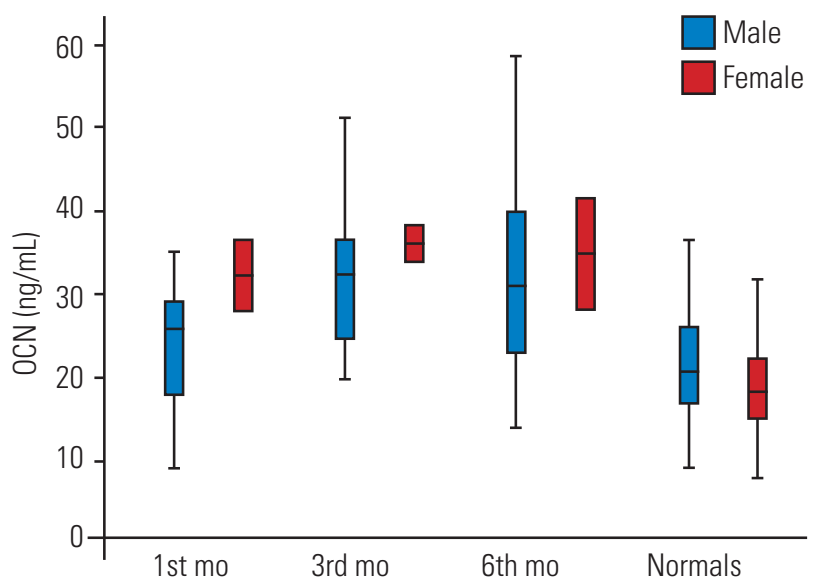

Fig. 2. Comparison of serum OCN levels among spinal cord injury population at 1 st 3 rd, 6 th month of injury with age- and gender-matched healthy controls. Values are given as box and whisker plots (minimum, 25, 50, 75 percentiles, and maximum). OCN, osteocalcin.

\section{Results}

Thirty patients fulfilling the study criteria were enrolled, and 20 patients completed the study. The study flowchart is shown in Fig. 1 and selected baseline characteristics of the study participants are shown in Table 1 . In this study, we included patients with AIS grading A, B, and C to obtain a uniform population of individuals who are most likely to suffer SCI-related bone loss. A total of $80 \%(n=16)$ of the population was AIS-A (motor and sensory complete), 5\% $(\mathrm{n}=1)$ AIS-B (motor complete and sensory incomplete), and $15 \%(n=3)$ AIS-C (motor and sensory incomplete). There was no statistically significant correlation between BTMs and the AIS grading, although patients with com-

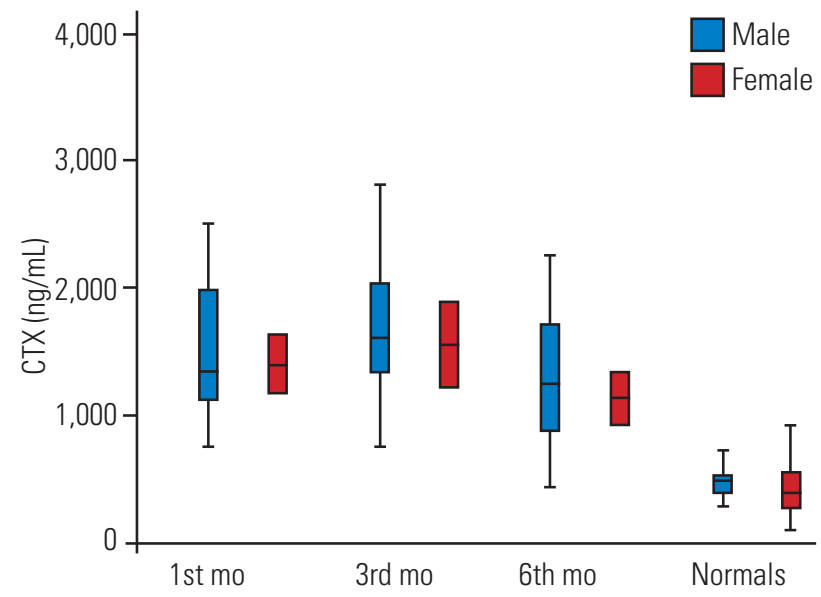

Fig. 3. Comparison of serum CTX levels among spinal cord injury population at $1 \mathrm{st}$, 3rd, 6th month of injury with age- and gender-matched healthy controls. Values are given as box and whisker plots (minimum, 25, 50, 75 percentiles, and maximum). CTX, C telopeptide.

plete SCI (AIS-A) had a highly raised level of the bone resorption marker CTX in comparison with those in the AIS-B and AIS-C groups. Serum calcium levels were within the normal range (8.3-10.4 mg/dL). PTH levels (normal range, $8-74 \mathrm{pg} / \mathrm{mL}$ ) were at the lower normal range, with the mean value being $15.2 \mathrm{pg} / \mathrm{mL}$. The mean $25(\mathrm{OH}) \mathrm{D}$ level among the study population was $14.9 \mathrm{ng} / \mathrm{mL}$ (normal range, $30-70 \mathrm{ng} / \mathrm{mL}$ ). Total alkaline phosphatase was marginally raised at 1 month after injury and was not significantly higher than the normative values.

The bone resorption marker serum CTX was significantly raised in comparison to a marginal rise in the bone formation marker (Figs. 2, 3). The maximum rise was observed at 3-4 months after SCI, following which there was 


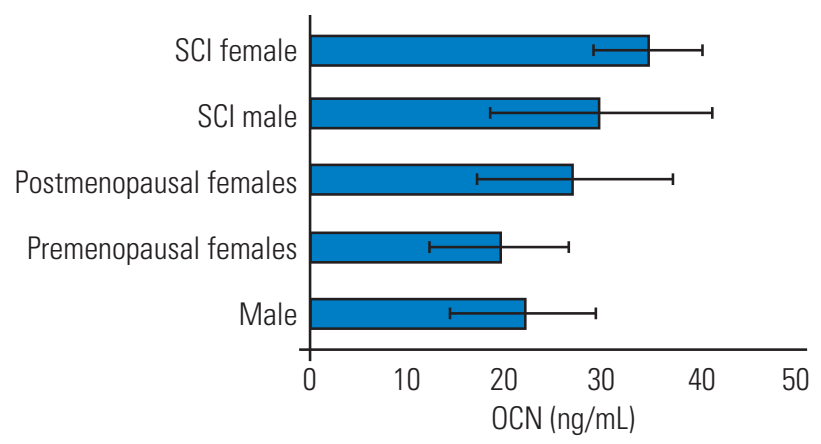

Fig. 4. Mean \pm standard deviation OCN levels $(\mathrm{ng} / \mathrm{mL}$ ) in healthy males, premenopausal females, postmenopausal females, SCI male, and SCI female population. OCN, osteocalcin; SCl, spinal cord injury.

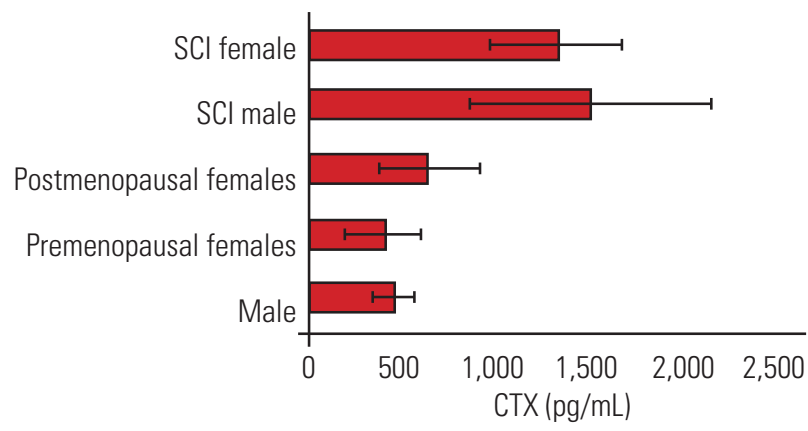

Fig. 5. Mean \pm standard deviation CTX levels $(\mathrm{pg} / \mathrm{mL})$ in healthy males, premenopausal females, postmenopausal females, SCI male, and SCI female population. CTX, C telopeptide; SCI, spinal cord injury.

a drop at the sixth month. The levels of the bone resorption marker serum CTX of SCI patients $(1,500.02 \pm 620.02$ $\mathrm{pg} / \mathrm{mL}$ ) were about 3 times higher than the mean values of CTX of the control population $(467 \pm 107 \mathrm{pg} / \mathrm{mL}$ male and $410 \pm 204.85 \mathrm{pg} / \mathrm{mL}$ premenopausal females) and about double that of postmenopausal females $(641 \pm 272.10$ $\mathrm{pg} / \mathrm{mL}$ ) (Figs. 4, 5).

\section{Discussion}

This is a prospective study of individuals with acute SCI evaluating the BTMs and comparing them with those of able-bodied males, premenopausal females, and a vulnerable group of postmenopausal females. In this study, the rate of BTMs was raised during the acute phase of SCI, and the rise in bone resorption markers was much greater than that of the bone formation markers. Bone resorption markers were significantly elevated in spinal injury patients in comparison to those in control males, and premenopausal and postmenopausal females.

Musculoskeletal changes following spinal injury are a result of irreversible neuroendocrinal changes in the body.
The sublesional skeletal system undergoes rapid remodeling, reflected in the increased rates of bone resorption and bone formation. In this study, we noticed that the bone resorption activity (indicated by serum CTX levels) was about 3 times higher than its normal upper limit. The rise in bone formation marker was also statistically significant, in comparison to the normal range, but there was a marked difference when compared with the rise in bone resorption marker. This indicates gross uncoupling of the remodeling cycle in favor of bone resorption, which can lead to loss of bone mass, resulting in the deterioration of bone microarchitecture and bone strength.

A significant rise in resorption markers was observed around 12-16 weeks, followed by a dip at the 24th week. Although there was a drop in serum BTMs at the 6th month, resorption markers remained significantly higher than normal values. In the literature, it is suggested that raised BTMs after acute SCI return toward the baseline by 6-24 months, with a maximum bone loss between 4 and 6 months after injury. Our findings are comparable to those in previous studies, in which BTMs remained elevated at 6 months following SCI [15]. Prolonged rapid bone remodeling in favor of bone resorption can affect the bone microarchitecture and bone quality. Thus, BTMs can be used either complementary to BMD or even independently in high risk group, such as SCI patients, for fracture risk assessment.

Bone loss of about $0.1 \%$ per week is observed during prolonged immobilization or microgravity conditions, whereas bone loss of $3 \%-5 \%$ per year is observed in postmenopausal females not taking bisphosphonates [16]. The rate of bone loss in SCI patients is substantially greater than that observed during immobilization, the postmenopausal phase, and other conditions vulnerable to bone loss. In this study, BTMs of age-matched male controls, premenopausal females, and postmenopausal females were compared with the mean values of BTMs in acute SCI patients (Tables 2,3). The levels of the bone resorption marker CTX were about 3 times higher than those in the control male and premenopausal female populations and double that of postmenopausal females. This was probably due to the loss of peripheral and central neurogenic influence on the sublesional skeletal tissue. Most studies involving BTMs were performed in postmenopausal females, while there is little literature on SCI patients. Studies in postmenopausal females have shown that increased resorption markers are associated with 
Table 2. Comparison of mean, $95 \%$ reference range of bone formation marker, serum osteocalcin, among control male, premenopausal women, postmenopausal women, and SCl patients

\begin{tabular}{|c|c|c|c|c|}
\hline \multirow{3}{*}{ Bone turnover marker } & \multicolumn{4}{|c|}{ Osteocalcin (ng/mL) } \\
\hline & \multirow{2}{*}{$\begin{array}{c}\text { Mean } \pm \text { standard } \\
\text { deviation }\end{array}$} & \multirow{2}{*}{$\begin{array}{l}95 \% \text { reference } \\
\text { range }\end{array}$} & \multicolumn{2}{|c|}{ Compared to SCI patients } \\
\hline & & & $p$-value & $95 \%$ confidence interval \\
\hline Premenopausal female & $19.42 \pm 7.14$ & 17.7 to 21.0 & $<0.001$ & -15.3 to -9.2 \\
\hline Postmenopausal female & $26.88 \pm 9.94$ & 24.6 to 29.1 & 0.01 & -8.3 to -1.3 \\
\hline Males & $21.68 \pm 7.54$ & 18.76 to 24.60 & $<0.001$ & -14.5 to -5.5 \\
\hline SCI patients & $30.04 \pm 10.82$ & 28.15 to 35.19 & & \\
\hline
\end{tabular}

$\mathrm{SCl}$, spinal cord injury.

Table 3. Comparison of mean, 95\% reference range of bone resorption marker, serum $\mathrm{C}$ telopeptide, among control male, premenopausal women, postmenopausal women, and SCI patients

\begin{tabular}{|c|c|c|c|c|}
\hline \multirow{3}{*}{ Bone turnover marker } & \multicolumn{4}{|c|}{ C telopeptide (pg/mL) } \\
\hline & \multirow{2}{*}{$\begin{array}{l}\text { Mean } \pm \text { standard } \\
\text { deviation }\end{array}$} & \multirow{2}{*}{$\begin{array}{l}95 \% \text { reference } \\
\text { range }\end{array}$} & \multicolumn{2}{|c|}{ Compared to SCI patients } \\
\hline & & & $p$-value & $95 \%$ confidence interval \\
\hline Premenopausal female & $410 \pm 204.85$ & 363.5 to 457.1 & $<0.001$ & $-1,236.1$ to -932.5 \\
\hline Postmenopausal female & $641 \pm 272.10$ & 578.9 to 703.2 & $<0.001$ & $-1,012.1$ to -694.5 \\
\hline Male & $467 \pm 107$ & 425.5 to 508.5 & $<0.001$ & $-1,265.9$ to -788.7 \\
\hline SCI patients & $1,494.3 \pm 629$ & 1,332 to 1,657 & & \\
\hline
\end{tabular}

SCl, spinal cord injury.

increased fracture risk, independent of BMD [17]. In this study, we compared SCI patients with postmenopausal females and found that the level of resorption marker in the SCI group was double that in postmenopausal females. This probably indicates the severity of bone loss during the acute phase of SCI. Raised BTMs indicate rapid bone loss and are currently used for individual fracture risk assessment in postmenopausal females and for monitoring osteoporosis treatment, but they are not yet used in high risk groups such as SCI patients $[18,19]$.

Acute SCI presents with a state of spinal shock, resulting in sublesional flaccid muscle tone. Reduced muscle tone and significant loss of muscle mass following acute SCI reduce the strain on the bones. Prolonged lack of loading, disuse, or immobilization during the acute stage probably down regulates the mechanosensory feedback at the cellular level (osteocytes), which is responsible for promoting bone formation and inhibiting resorption-promoting agents such as sclerostin [20]. These factors all together probably aggravate the bone loss by rapidly upregulating resorption activators during the acute phase of SCI.

The mean value of serum PTH level among these pa- tients was $15.2 \mathrm{ng} / \mathrm{dL}$. Only four patients had PTH values $>20 \mathrm{ng} / \mathrm{dL}$. The lower distribution of PTH levels among complete SCI patients was probably due to elevated ionized serum calcium levels and due to the mobilization of skeletal calcium as a result of SCI-related immobilization or disuse-related bone loss. Suppressed PTH levels reduce PTH-mediated tubular absorption of calcium, leading to hypercalciuria [21]. Studies have shown that PTH remains suppressed for about 1-4 months post-SCI and then returns toward the normal range $[22,23]$. In this study, no correlation was obtained between the BTM and PTH levels, which probably suggests a significant role of nonhormonal factors that contribute to rapid bone remodeling and bone loss.

Most of the patients $(\mathrm{n}=16)$ had vitamin D levels $<20$ $\mathrm{ng} / \mathrm{dL}$ and were started on oral cholecalciferol supplementation. Our results were consistent with previous studies, as no association was observed between vitamin $\mathrm{D}$ and BTMs. Reliable dietary details were not obtained, so it is difficult to comment on the effect of vitamin D on BTMs. Currently, there is no evidence to suggest that vitamin D correction to therapeutic levels may prevent bone loss fol- 
lowing SCI [24].

Osteoporosis is considered to be a major complication in the SCI population. Currently, there are no clinical guidelines for the early diagnosis, prevention, treatment, or reversal of SCI-related osteoporosis and hence there are wide variations in treatment practices [25]. Fracture risk assessment in the SCI population should identify SCI-specific risk factors. Non-modifiable SCI-specific risk factors include age, peak bone mass, duration of injury, neurological level, motor and sensory complete injury. The rate of bone remodeling, $\mathrm{BMD}$, hypovitaminosis $\mathrm{D}$, smoking, appropriate wheelchair transfer skills, medications such as opioids, bbenzodiazepines, and their link with falls are the modifiable SCI-specific risk factors [12]. Bone strength comprises BMD and bone quality [26]. BMD is a static measurement and easily measurable by DEXA scanning, whereas bone quality, which depends on material composition and structural design, is difficult to assess in clinical settings, except the BTMs, which represent bone remodeling [26,27]. The World Health Organization defines osteoporosis by means of BMD, but this has its own limitations $[11,14]$. Firstly, detectable changes in bone density become apparent at 6-12 months after SCI and hence the phase of maximal bone loss (i.e., 3-6 months) is often underestimated and therapeutic intervention is delayed $[6,28]$. Significant changes in BTMs may be appreciable at as early as $2-3$ months of treatment, whereas detectable changes in bone density can take up to 2 years to become apparent [29]. Secondly, there is evidence that changes in BMD do not correlate with reduction of risk of fracture and fracture incidence upon treatment with bisphosphonates [30]. The use of antiresorptive agents for osteoporosis correlates with a significant reduction in remodeling rates, rather than an increase in BMD. BTMs have been useful for measuring the response to pharmacotherapy in postmenopausal females, resulting in improved patient compliance [31]. BTMs being dynamic metabolic responders can be used during the acute phase and followup investigations for fracture risk assessment and clinical decision-making for pharmacological management in patients with SCI. Hence, both BMD and BTMs together can complement each other and better prognosticate the fracture risk, which can aid in the clinical management of bone health in the SCI population. Further studies using BTMs and BMD together are required to assess the utility of both of these parameters, in terms of early detection, and monitoring bone loss and response to pharmacother- apy in the acute and chronic SCI population.

The disproportionate increase in bone resorption markers suggests a hyper-resorptive bone state following acute SCI. Altered bone microarchitecture and reduced bone strength following SCI predispose these patients to lowtrauma fractures. According to previous studies, 15\%$40 \%$ of individuals with traumatic SCI sustain fractures of the lower extremities $[2,32]$. Other than completeness of SCI, no factors such as age, neurological level, duration of injury, and mode of ambulation correlated with pathological fractures following SCI [2]. Hence, we should target modifiable factors such as vitamin D deficiency, raised BTMs, and low BMD, all of which used together judiciously can help in proper fracture risk assessment and management of osteoporosis.

Uniform population sampling was challenging because of multiple varying parameters such as the neurological level of injury, AIS grading, muscle tone, duration of mechanical loading, and participation in mobilization activity. To avoid heterogeneity of the population, SCI patients with quadriplegia were not included in this study. All of the participants continued the routine therapy during their stay in the rehabilitation center. In our study, the source of potential bias was the varying degrees of mobilization (wheelchair mobilization/ambulation with orthosis) and duration of mechanical loading.

Our study confirms earlier findings on BTMs in patients with SCI. Unlike DEXA scanning, BTMs help in the early detection of bone loss following SCI, which provides us with the opportunity to monitor bone loss, assess fracture risk, and plan pharmacological intervention. The strength of this study was its prospective design, focus on the acute phase of SCI, and uniform population characteristics, including only paraplegic population. The main limitation of this study was its small sample size, with a predominantly male population. This can be explained by the fact that, in developing countries like India, males are four times more likely to be affected by SCI than females [33]. Moreover, this study had a short duration, in which BTMs were evaluated in the study subjects at baseline, and 3 and 6 months, whereas the control population had only single baseline values.

\section{Conclusions}

Longitudinal observation of BTMs in patients with acute SCI demonstrated a significant rise in the bone resorp- 
tion marker CTX, whereas the rise in the bone formation marker OCN was marginal. Persistent and markedly elevated bone resorption markers indicate rapid ongoing bone loss during the acute phase of spinal injury. Unlike DEXA scanning, BTMs may aid in the early detection and evaluation of bone loss. Bone health assessment and monitoring should begin early during the acute phase of SCI rehabilitation.

\section{Conflict of Interest}

No potential conflict of interest relevant to this article was reported.

\section{References}

1. Jiang SD, Jiang LS, Dai LY. Spinal cord injury causes more damage to bone mass, bone structure, biomechanical properties and bone metabolism than sciatic neurectomy in young rats. Osteoporos Int 2006;17:1552-61.

2. Gifre L, Vidal J, Carrasco J, et al. Incidence of skeletal fractures after traumatic spinal cord injury: a 10-year follow-up study. Clin Rehabil 2014;28:361-9.

3. Leblanc AD, Schneider VS, Evans HJ, Engelbretson DA, Krebs JM. Bone mineral loss and recovery after 17 weeks of bed rest. J Bone Miner Res 1990;5:84350.

4. Jiang SD, Jiang LS, Dai LY. Mechanisms of osteoporosis in spinal cord injury. Clin Endocrinol (Oxf) 2006;65:555-65.

5. Bonewald LF, Johnson ML. Osteocytes, mechanosensing and Wnt signaling. Bone 2008;42:606-15.

6. Maimoun L, Couret I, Mariano-Goulart D, et al. Changes in osteoprotegerin/RANKL system, bone mineral density, and bone biochemicals markers in patients with recent spinal cord injury. Calcif Tissue Int 2005;76:404-11.

7. Frotzler A, Berger M, Knecht H, Eser P. Bone steadystate is established at reduced bone strength after spinal cord injury: a longitudinal study using peripheral quantitative computed tomography (pQCT). Bone 2008;43:549-55.

8. Dudley-Javoroski S, Shields RK. Regional cortical and trabecular bone loss after spinal cord injury. J Rehabil Res Dev 2012;49:1365-76.

9. Coupaud S, McLean AN, Purcell M, Fraser MH, Al- lan DB. Decreases in bone mineral density at cortical and trabecular sites in the tibia and femur during the first year of spinal cord injury. Bone 2015;74:69-75.

10. Hartkopp A, Murphy RJ, Mohr T, Kjaer M, BieringSorensen F. Bone fracture during electrical stimulation of the quadriceps in a spinal cord injured subject. Arch Phys Med Rehabil 1998;79:1133-6.

11. Garg MK, Kharb S. Dual energy X-ray absorptiometry: pitfalls in measurement and interpretation of bone mineral density. Indian J Endocrinol Metab 2013;17:203-10.

12. Cervinka T, Lynch CL, Giangregorio L, et al. Agreement between fragility fracture risk assessment algorithms as applied to adults with chronic spinal cord injury. Spinal Cord 2017;55:985-93.

13. Maynard FM Jr, Bracken MB, Creasey G, et al. International standards for neurological and functional classification of spinal cord injury. American Spinal Injury Association. Spinal Cord 1997;35:266-74.

14. Shetty S, Kapoor N, Dian Bondu J, Antonisamy B, Thomas N, Paul TV. Bone turnover markers and bone mineral density in healthy mother-daughter pairs from South India. Clin Endocrinol (Oxf) 2016;85:725-32.

15. Reiter AL, Volk A, Vollmar J, Fromm B, Gerner HJ. Changes of basic bone turnover parameters in shortterm and long-term patients with spinal cord injury. Eur Spine J 2007;16:771-6.

16. Recker R, Lappe J, Davies K, Heaney R. Characterization of perimenopausal bone loss: a prospective study. J Bone Miner Res 2000;15:1965-73.

17. Garnero P, Sornay-Rendu E, Chapuy MC, Delmas PD. Increased bone turnover in late postmenopausal women is a major determinant of osteoporosis. J Bone Miner Res 1996;11:337-49.

18. Wheater G, Elshahaly M, Tuck SP, Datta HK, van Laar JM. The clinical utility of bone marker measurements in osteoporosis. J Transl Med 2013;11:201.

19. Tarantino U, Iolascon G, Cianferotti L, et al. Clinical guidelines for the prevention and treatment of osteoporosis: summary statements and recommendations from the Italian Society for Orthopaedics and Traumatology. J Orthop Traumatol 2017;18(Suppl 1):3-36.

20. Baron R, Kneissel M. WNT signaling in bone homeostasis and disease: from human mutations to treatments. Nat Med 2013;19:179-92.

21. Stewart AF, Adler M, Byers CM, Segre GV, Broadus 
AE. Calcium homeostasis in immobilization: an example of resorptive hypercalciuria. N Engl J Med 1982;306:1136-40.

22. Kostovski E, Hjeltnes N, Eriksen EF, Kolset SO, Iversen PO. Differences in bone mineral density, markers of bone turnover and extracellular matrix and daily life muscular activity among patients with recent motor-incomplete versus motor-complete spinal cord injury. Calcif Tissue Int 2015;96:145-54.

23. Hummel K, Craven BC, Giangregorio L. Serum $25(\mathrm{OH}) \mathrm{D}, \mathrm{PTH}$ and correlates of suboptimal $25(\mathrm{OH})$ $D$ levels in persons with chronic spinal cord injury. Spinal Cord 2012;50:812-6.

24. Bauman WA, Emmons RR, Cirnigliaro CM, Kirshblum SC, Spungen AM. An effective oral vitamin D replacement therapy in persons with spinal cord injury. J Spinal Cord Med 2011;34:455-60.

25. Troy KL, Morse LR. Measurement of bone: diagnosis of SCI-induced osteoporosis and fracture risk prediction. Top Spinal Cord Inj Rehabil 2015;21:267-74.

26. Armas LA, Recker RR. Pathophysiology of osteoporosis: new mechanistic insights. Endocrinol Metab Clin North Am 2012;41:475-86.

27. Seeman E, Delmas PD. Bone quality: the material and structural basis of bone strength and fragility. $\mathrm{N}$
Engl J Med 2006;354:2250-61.

28. Paker N, Bugdayci D, Ersoy S, Uysal E, Elbirlik S. Bone loss and bone turnover in acute and chronic spinal cord injured patients. Neurosciences (Riyadh) 2007;12:232-5.

29. Lodder MC, Lems WF, Ader HJ, et al. Reproducibility of bone mineral density measurement in daily practice. Ann Rheum Dis 2004;63:285-9.

30. Watts NB, Geusens P, Barton IP, Felsenberg D. Relationship between changes in BMD and nonvertebral fracture incidence associated with risedronate: reduction in risk of nonvertebral fracture is not related to change in BMD. J Bone Miner Res 2005;20:2097104.

31. Garnero P. Biomarkers for osteoporosis management: utility in diagnosis, fracture risk prediction and therapy monitoring. Mol Diagn Ther 2008;12:157-70.

32. Akhigbe T, Chin AS, Svircev JN, et al. A retrospective review of lower extremity fracture care in patients with spinal cord injury. J Spinal Cord Med 2015;38:29.

33. Birua GJS, Munda VS, Murmu NN. Epidemiology of spinal injury in North East India: a retrospective study. Asian J Neurosurg 2018;13:1084-6. 